\title{
In-Lens Cryo-High Resolution Scanning Electron Microscopy: Methodologies For Molecular Imaging Of Self-Assembled Organic Hydrogels
}

\author{
R.P. Apkarian, ${ }^{1 *}$ E.R. Wright,,${ }^{1,2,4}$ V.A. Seredyuk, ${ }^{2}$ S. Eustis, ${ }^{3}$ L. A. Lyon, ${ }^{3}$ \\ V.P. Conticello, ${ }^{2}$ F.M. Menger, ${ }^{2}$ \\ ${ }^{1 *}$ Integrated Microscopy \& Microanalytical Facility, Emory University, Atlanta, GA 30322 \\ ${ }^{2}$ Department of Chemistry, Emory University, Atlanta, GA 30322 \\ ${ }^{3}$ School of Chemistry \& Biochemistry, Georgia Institute of Technology, Atlanta, GA 30332 \\ ${ }^{4}$ Department of Materials Science \& Engineering, University of Southern California, Los Angeles, \\ CA 90089
}

Cryo-etch HRSEM represents an essential analytical tool for quality control and development of biological and organic hydrogels. Composed of particles, fibers and filaments hydrogels are selfassembled material systems useful for numerous biomedical applications. We describe the gross and fine morphology of three different self-assembled organic hydrogels using cryo-etch (in-lens) high resolution FESEM in an effort to understand the forces involved in gel formation. The first gel, a gemini-surfactant $(\mathrm{gS})$ forms a concentration dependent porous high-density network. Low temperature imaging using an in-lens FESEM rapidly assessed the gS gel matrix, revealed after etching away vitreous ice [1]. Similarly, $210 \mathrm{~nm}$ poly N-isopropyl acrylamide (pNIP Am) spheres self assemble upon centrifugation into an iridescent hydrogel below its transition temperature [2]. A triblock elastin hydrogel self assembles above its inverse temperature transition point producing a biomaterial with controllable bio-erosion characteristics [3].

Hydrogels were loaded into gold planchets (Balzers, 012 0130T) and equilibrated at their gelation temperature then ethane plunge or high presser frozen (Balzers, HPF-010). A planchet was loaded onto the Gatan CT3500 cold stage under $\mathrm{LN}_{2}$, the specimen is fractured with a prechilled blade, then the dual shutters were closed while being rinsed with $\mathrm{LN}_{2}$. The cryostage was transferred to the Denton DV-602 Chromium coater and immediately evacuated to $2 \times 10^{-7}$ torr. The specimen temperature was ramped from $-170^{\circ} \mathrm{C}$ to $-105^{\circ} \mathrm{C}$ for etching times between $2-10$ min depending on the hydration forces within the sample, and returned to $\left(-170^{\circ} \mathrm{C}\right)$ for sputter deposition of a $1-2 \mathrm{~nm}$ $\mathrm{Cr}$ film [4]. Images were digitally recorded from the in-lens DS-130 FESEM / Gatan cold stage at $115^{\circ} \mathrm{C}$.

Electrostatic and van der Waals forces, and in the case of elastin protein, hydrogen bonding, together regulate the gel structure and its hydration shell. The etching time necessary to remove bulk frozen water and reveal the gel fine structure was indicative of these forces. A $5 \% g \mathrm{gS}\left(\mathrm{C}_{30} \mathrm{H}_{64} \mathrm{NO}_{4} \mathrm{P}\right)$ sample prepared by HPF and etched for 5 min revealed a highly porous interwoven filamentous network (Fig.1). The morphology of p-NIP Am gels etched for 2 min revealed $200 \mathrm{~nm}$ bead cores with an extensive lace network composed of polyelectrolyte chains (Fig. 2). Several genetically engineered triblock elastin polymers have been analyzed by cryo-etch HRSEM. The fine filamentous honeycomb motif of an elastin triblock hydrogel was recorded from a 2.5 min etched sample (Fig. 3). Cryoetch HRSEM accurately provides fine filamentous and particulate details in the 1-10 nm range without crosslinking and drying. Hydrogels are highly hydrated, and like cells retain their skeletal-like architecture when rapidly cryo-immobilized, followed by vacuum sublimation of ice, thin metal coating and low temperature imaging in-lens of a FESEM. [5] 


\section{References}

[1] F. M. Menger et al., J. Am. Chem. Soc. 124 (2002) 12408.

[2] J.D. Debord et al., Adv. Mater. 14 (2002) 658.

[3] E.R. Wright et al., Adv. Funct. Mater. 12 (2002) 149.

[4] E.R. Wright et al., Microsc. Microanal. 9 (2003) in press.

[5] This research was supported by NIH grant RR14717-01A1.

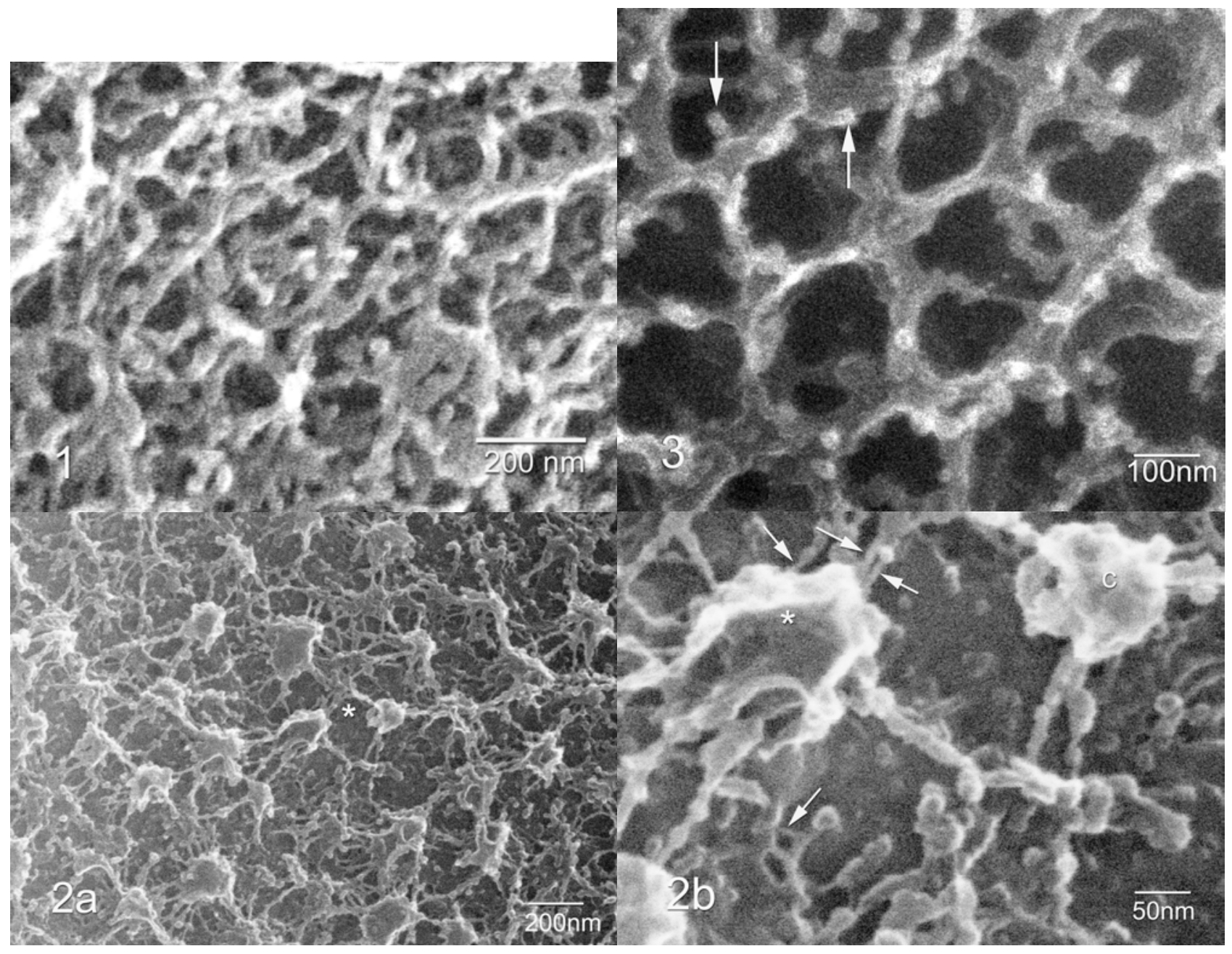

FIG. 1. Cryo-etch HRSEM of Gemini surfactant [5\%] high-pressure frozen and etched 5 min.

FIG. 2. Cryo-etch (2-min) of pNIP Am. (a) note cores and polyelectrolyte chains, * area seen in 2 b. (b) Intact particle core (c) and (*) fractured core chained together by $\sim 5 \mathrm{~nm}$ thin filaments (arrows). FIG. 3. Triblock elastin hydrogel, $2.5 \mathrm{~min}$ etch. $20 \mathrm{~nm}$ fibers (arrows) woven into honeycomb. 